\title{
Gerbes and Massive Type IIA
}

\author{
Jussi Kalkkinen* \\ SISSA, via Beirut 4, Trieste 34014, Italy \\ INFN, Sezione di Trieste \\ E-mail: kalkkine@sissa.it
}

ABstract: We discuss certain type IIA supergravity solutions, and the global structures associated to them. We review a global formalism for theories that involve massive tensor fields in general, and apply it in these special cases.

\section{KEYwORDS: 'S̄uperstring Vacua, Differential and Ālgebraic Geometry, p-branes'}

\section{Introduction}

Studying the Physics that underly the NeuveuSchwarz two-form potential, the $B$-field, seems to be a fruitful way of incresing our understanding of nonperturbative structures in string theory. This particular field appears in many different contexts:

- NS5-branes are magnetically charged with respect to it. The classical limit of the world-volume theories realized on coinciding type IIA NS5-branes is still poorly understood, as it involves a self-dual variant of the $B$-field.

- A flat $B$-field, or at least torsion, is still necessary for the classification of D-brane charges in K-theory.

- The $B$-field enters the discussion of anomalies in world-sheet $\sigma$-models in an interesting way.

- In the presence of a constant $B$-field the effective world-volume theories on D-branes can be described in terms noncommutative geometry.

All of these appearances are somewhat related. As argued in Refs. [1] seem to yield to a description in terms of gerbes

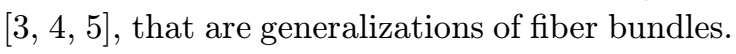

*This work was supported by the European Union TMR program CT960045.
In this article we shall discuss some aspects of these structures, and give concrete examples in terms of type II supergravity solutions.

\section{Massive type IIA supergravity}

The effective low-energy theory of the type IIA string is the type IIA supergravity. The type IIA string theory includes a ten-form field strength, which can be treated by Hodge duality as a cosmological constant. If this gauge field is excited, the correct low energy theory is the massive deformation of the type IIA supergravity. Its action that was first discovered by Romans [6] in

$$
\begin{aligned}
S= & \int \mathrm{e}^{-2 \phi}\left(* R+4 \mathrm{~d} \phi \wedge * \mathrm{~d} \phi-\frac{1}{2} G \wedge * G\right) \\
& -\frac{1}{2} R(C) \wedge * R(C) \\
& +\frac{1}{2} \mathrm{~d}^{-1}\left(R^{[4]} \wedge R^{[4]} \wedge G\right) \\
& +(\text { fermions }) .
\end{aligned}
$$

The only non-trivial components of $R^{[2 k]}$ are those for $2 k=0,2,4$. These modified field strengths can be summarized in the expansion

$$
R(C)=\mathrm{d} C-G \wedge C+m \mathrm{e}^{B},
$$

where $B$ is the NS two-form, $G=\mathrm{d} B$, and $m$ the mass parameter.

This action possesses various gauge symmetries. In order to isolate the essential features in these symmetries, let us concentrate on the 
trasformation properties of the RR one-form potential $C^{[1]}$. This field looks like a U(1)-gauge field, as we are allowed to shift it (in a suitable background of other fields) by any closed form. Geometrically this means that we could try to interpret it as a connection on a line bundle $\mathcal{L}$. Then the Chern class of this bundle $c_{1}(\mathcal{L})$ would just be the D6-brane charge. However, we are also allowed to shift it

$$
C^{[1] \prime}=C^{[1]}+m \eta
$$

using any one-form $\eta$. This symmetry can be used to gauge away the one-form RR-potential completely, after which the part of the Lagrangian that involves the $B$-field is

$$
\int\|\mathrm{d} B\|^{2}+\frac{1}{2} m^{2}\|B\|^{2}+\ldots
$$

In this way the $B$-field becomes a massive tensor field, thus absorbing the together with the RR-field disappearing degrees of freedom. In the presence of D0 or D6-branes this interpretation might seem problematic at the first sight, as eliminating the RR-field would seem to violate charge conservation. Indeed, the system is actually also invariant under shifts of $\mathrm{d} C^{[1]}$ by any closed form. If there is a topologically nontrivial two-cocycle in the cohomology of the space-time, we can use this symmetry to shift the D6-brane charge associated naively to the bundle $\mathcal{L}$ arbitrarily.

The physical field strengths are really the modified field strengths instead, and the bare RR-fields have no independent meaning. The above example raises the question, however, of how to interpret the RR one-forms geometrically and what really is their relation to the NS twoform.

All of this is naturally also consistent with the fact that D-brane charges are indeed know to be classified rather in terms of K-theory than directly in terms of the cohomoly of the RR-fields $[\overline{7}]$. However, this classification is completely understood only for $G=0$. What happens for $G \neq 0$ ? In order to attempt to answer these question we seem to have to consider generalized line bundles, gerbes.

\section{Some tools}

\subsection{Line bundles}

Let us briefly recapitulate the information that is needed to build a line bundle. To start with, we need the projection $\pi: \mathcal{L} \longrightarrow M$ form the full space to the base space. We also need to know how the fibers $\mathrm{U}(1) \cong V_{\alpha}=\pi^{-1}(p), p \in \mathcal{U}_{\alpha} \subset M$ transform when we move from one open neighbourhood $\mathcal{U}_{\alpha}$ of the base space $M$ to another, $\mathcal{U}_{\beta}$. This information is encoded in the U(1)-valued transition functions $g_{\alpha \beta}$.

Two consistency conditions ensue: First, $g$ must be antisymmetric in the sence that $g_{\alpha \beta}=$ $g_{\beta \alpha}^{-1}$ and, second, $\delta g_{\alpha \beta \gamma}=1$ where $\delta g$ is shorthand for

$$
g_{\beta \gamma} g_{\alpha \gamma}^{-1} g_{\alpha \beta} .
$$

Given the base space, the collection of these transition functions essentially defines the line bundle.

Not all line bundles defined this way are inequivalent: one can always deform the transitions functions $g_{\alpha \beta}$ multiplying them with any $\mathrm{U}(1)$ valued functions of the form $h_{\alpha} h_{\beta}^{-1}$ without changing the topology of the bundle. These deformations are nothing but gauge transformations. A convenient way of classifying these bundles is available, if we have a connection $\nabla=$ $\mathrm{d}+A$ on $\mathcal{L}$. Then the first Chern class

$$
c_{1}(\mathcal{L})=\left[\frac{\mathrm{d} A_{\alpha}}{2 \pi}\right] \in H^{2}(M, \mathbb{Z})
$$

distinguishes between inequivalent bundles in terms of the cohomology of the base space $M$.

\subsection{Gerbes}

Gerbes are formal generalizations of line bundles in the sense that, given the base space $M$, they are (equivalence classes of) collections of U(1)valued functions $g_{\alpha \beta \gamma}$ on each triple intersection $\mathcal{U}_{\alpha} \cap \mathcal{U}_{\beta} \cap \mathcal{U}_{\gamma}$. Also these functions must be antisymmetric and satisy $\delta g_{\alpha \beta \gamma \delta}=1$, shorthand for

$$
g_{\beta \gamma \delta} g_{\alpha \gamma \delta}^{-1} g_{\alpha \beta \delta} g_{\alpha \beta \gamma}^{-1} \text {. }
$$

Given a collection of functions $h_{\alpha \beta}$ on each double intersection we can shift $g$ by

$$
g_{\beta \gamma \delta}^{\prime}=g_{\beta \gamma \delta} \delta h_{\beta \gamma \delta},
$$


where $\delta h_{\beta \gamma \delta}=h_{\gamma \delta} h_{\beta \delta}^{-1} h_{\beta \gamma}$. Identifying $g \sim g^{\prime}$ we get a class in the second Čech -cohomology group.

It is useful to equip the gerbe as defined above with some additional structure. In the following we shall consider a gerbe $\left\{g_{\beta \gamma \delta}\right\}$ with curving $G \in H^{3}(M, \mathbb{Z})$ and connective structure. Concretely, we need the following data:

$g_{\alpha \beta \gamma}$ A Čech -cocycle.

$A_{\alpha \beta}$ connections on a line bundles $\mathcal{L}_{\alpha \beta}$ defined over the two-fold intersections $\mathcal{U}_{\alpha} \cap \mathcal{U}_{\beta}$.

$B_{\alpha}$ A locally defined two-form potential of $G=$ $\mathrm{d} B_{\alpha}$.

These differential forms are useful for characterizing inequivalent gerbes and, as we shall presently see, they are also the fields that would appear in a supergravity realization of a gerbe.

The forms $A_{\alpha \beta}$ and $B_{\alpha}$ satisfy the following consistency conditions

1) $\mathcal{L}_{\alpha \beta}=\mathcal{L}_{\beta \alpha}^{-1}$.

2) $\mathcal{L}_{\alpha \beta} \otimes \mathcal{L}_{\beta \gamma} \otimes \mathcal{L}_{\gamma \alpha}$ trivial where defined.

3) $B_{\alpha}-B_{\beta}=\mathrm{d} A_{\alpha \beta}$ where defined.

Here the Čech -cocycle does not appear explicitly any more, though the information of its topological class is contained in the equations.

Let us consider the trivial situation first: If there were bundles $\mathcal{L}_{\alpha}$ that satisfy $\mathcal{L}_{\alpha} \otimes \mathcal{L}_{\beta}^{-1}=$ $\mathcal{L}_{\alpha \beta}$ then we could write

$$
B_{\alpha}+c_{1}\left(\mathcal{L}_{\alpha}\right)=B_{\beta}+c_{1}\left(\mathcal{L}_{\beta}\right)
$$

Identifying the $B_{\alpha}=m B_{\mathrm{NS}}$ and $c_{1}\left(\mathcal{L}_{\alpha}\right)=\mathrm{d} C_{\mathrm{RR}}^{[1]}$ this condition just reduces to the statement that

$$
R^{[2]}=\mathrm{d} C_{\mathrm{RR}}^{[1]}+m B_{\mathrm{NS}}
$$

is a well defined gauge invariant quantity.

\section{Solutions}

In the following we will consider as in Ref. [1] massive type IIA supergravity on a space time $M^{1,5} \times \mathbb{R} \times S^{3}$, where $M^{1,5}$ is an arbitrary manifold. Our constructions are analogues of Dirac's treatment of a monopole, though one dimension higher. Here NS5-branes with world-volumes along $M^{1,5}$ play the role of the monopole sources, located at the extracted point in $\mathbb{R}^{4 *} \cong \mathbb{R} \times S^{3}$. The Dirac string will turn out to be the D6-brane.

The strategy of solving the equations of motion is the following: We show here that the various charges are conserved and that all fields are well defined. This amounts to solving the formfield Bianchi identities and equations of motion. The metric and the dilaton fields will adapt when these sources are consistently put in place. The actual solutions can be described most conveniently in the T-dual type IIB description (for this, see Ref. [鱼]

\subsection{D6-branes ending on NS5-branes}

In the spirit of the Dirac monopole bundle, we always cover the three-sphere with two open charts $\mathcal{U}_{0}$ and $\mathcal{U}_{1}$, and study the behaviour of various supergravity fields on their intersection. Choose now a fixed point $p \subset S^{3}$ from the sphere outside $\mathcal{U}_{0}$. Let $H$ satisfy $^{1}$

$$
\Delta H=[V]-2 \pi \delta_{p} \in H^{3}\left(S^{3}, \mathbb{Z}\right),
$$

where $[V]$ is the de Rham class of the volume form i.e. generator of $H^{3}\left(S^{3}, \mathbb{Z}\right), \delta_{p}$ is the Poincaré dual of the point $p, Q$ is a parameter, and $\Delta=$ $\left\{\mathrm{d}, \mathrm{d}^{\dagger}\right\}$ is the Hodge-de Rham Laplacian. On the coordinate patch $\mathcal{U}_{1}$ we can similarly define

$$
\Delta H_{1}=[V] .
$$

The ansatz for the NS two-form is locally

$$
B=\left\{\begin{array}{ll}
Q \mathrm{~d}^{\dagger} H & \text { on } \mathcal{U}_{0} \\
Q \mathrm{~d}^{\dagger} H_{1} & \text { on } \mathcal{U}_{1}
\end{array} .\right.
$$

The field strength is

$$
G=\mathrm{d} B=Q[V],
$$

which yields the NS5-brane charge $2 \pi Q$. Since $[G / 2 \pi]$ is in integral cohomology, $Q$ must be an integer.

For generality we consider the mass parameter in Romans' supergravity as a local constant

$$
m=\left\{\begin{array}{lll}
m_{0} & \text { on } \mathcal{U}_{0} \\
m_{1} & \text { on } \mathcal{U}_{1}
\end{array} .\right.
$$

\footnotetext{
${ }^{1}$ The present ansatz makes use of a construction in [5i5].
} 
On the intersection of charts $\mathcal{U}_{0} \cap \mathcal{U}_{1}$ the charge is the difference in cosmological constants $q(D 8)=$ $m_{1}-m_{0}=\Delta m$.

We shall further assume

$$
\mathrm{d} C_{\mathrm{RR}}^{[1]}= \begin{cases}0 & \text { on } \mathcal{U}_{0} \\ Q \mathrm{~d}^{\dagger}\left(m_{0} H_{0}-m_{1} H_{1}\right) & \text { on } \mathcal{U}_{1}\end{cases}
$$

Now $R^{[2]}$ turns out to be a globally defined differential form, as becomes a physical field strength.

\subsection{Charge quantization}

The D6-brane charge can be calculated integrating over the charge density on a disc $D \subset \mathcal{U}_{1}$

$$
\begin{aligned}
q_{\mathrm{D} 6}= & \left(m_{0}-m_{1}\right) Q \operatorname{vol}(\partial D) \\
& -2 \pi m_{0} Q .
\end{aligned}
$$

We want to interpret the restriction of the nongauge invariant two-form $\mathrm{d} C^{[1]}$ on $\mathcal{U}_{0} \cap \mathcal{U}_{1}$ as the curvature $F$ of a suitable line bundle on the intersection. If this can be done the whole structure extends to a gerbe. The base space of the bundle $\mathcal{L}_{01}$ we are looking for is topologically a cylinder. The condition for $\mathcal{L}_{01}$ to be an on the cylinder well defined line bundle is that the charge $q_{\mathrm{D} 6}$ be quantized. As the factor $\operatorname{vol}(\partial D)$ in the formula for the flux depends continuously on the choice of $D^{3}$, we are lead to the quantization conditions

$$
\begin{aligned}
m_{0} & =m_{1} \\
m_{0} Q & \in \mathbb{Z} .
\end{aligned}
$$

The first one implies that a D8-brane would separate two different gerbes from one another other. As noted above, $Q$ is an integer. This leads to the quantization of the cosmological constant as well, which is consistent with the results of Ref. [1].

\subsection{Domain walls}

Another instructive example is that of a domain wall, where $m_{0} \neq m_{1}$. This configuration will describe the boundary between two different gerbes separated by the wall. Let us in particular consider the above computation with the modification

$$
\Delta H=[V]
$$

_on $\mathcal{U}_{0}$ _as_well. . Taking_the limit $\mathcal{U}_{1-} \ldots\{$ pt\}_ we _ _ _ [1] J. Kalkkinen, '

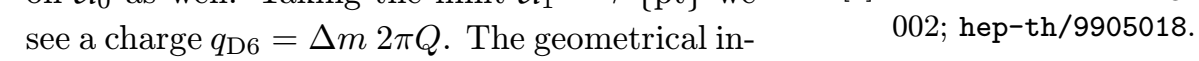

terpretation for this is that there are $\Delta m Q$ copies of D6-branes ending on a NS5-branes located on a D8-brane domain wall.

\section{Holonomies}

It is physically reasonable to be interested in a description of these systems in terms of transition functions $g_{\alpha \beta}$, or their equivalents in gerbes $g_{\alpha \beta \gamma}$, because they are directly related to holonomies. The connection on a line bundle, and the one and the two-form fields on a gerbe, give tools to calculate them effectively as was done, for instance, in the treatment of world-sheet anomalies in Ref. [9in]. One of the intriguing but still open problems is to find the classical limit of coinciding NS5-branes in type IIA, cf. Refs. [1] 슨. In Ref. [2in] a non-Abelian generalization of these structures was suggested, where the interplay of the holonomies of local Chan-Paton bundles and the characteristic class of a gerbe was crucial.

\section{Conclusions}

We have reviewed a class of solutions of type IIA supergravity that involve NS5-branes and D6branes. These configurations seem to defy the traditional description in terms of global bundles over submanifolds, and should be described in terms of gerbes. Configurations of this kind appear generically in any theory that involves massive tensor fields, and in particular in supergravity in the presence of NS5-branes.

It seems reasonable to expect that the methods reviewed here might be instrumental in studying consistency conditions for D-brane embeddings in nontrivial $B$-field backgrounds, as well as in understanding the classical limit of coinciding NS5-branes. Also the classification problem of D-branes in the presense of a topologically nontrivial $B$-field seems to involve classification of gerbes as a natural extension of the K-theory classification of fiber bundles over the branes.

\section{References}

[2] J. Kalkkinen, hep-th/9910048'. 
[3] J. Giraud, "Cohomologie Non-Abélienne," (Springer-Verlag, Berlin 1971)

[4] J.-L. Brylinski, "Loop Spaces, Characteristic Classes and Geometric Quantization,"

(Birkäuser, Boston 1993)

[5] N. Hitchin, math/9907034.

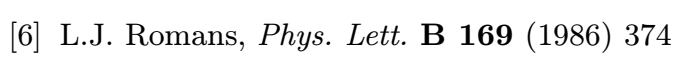

[7] E. Witten, 'J. High Energy Phys. 12 (1998) 019;; hep-th/9810188.

[8] M.B. Green, C.M. Hull and P.K. Townsend, Phys. Lett. B 382 (1996) 655ep-th/9604119;

[9] D.S. Freed and E. Witten, hep-th/9907189!

[10] E. Witten, IJ. Geom. Phys. 22 (1997) 103; hep-th/9610234!

[11] X. Bekaert, M. Henneaux and A. Sevrin, 'Phys. Lett. B $468(1999) 228$; ' hep-th/9909094! 